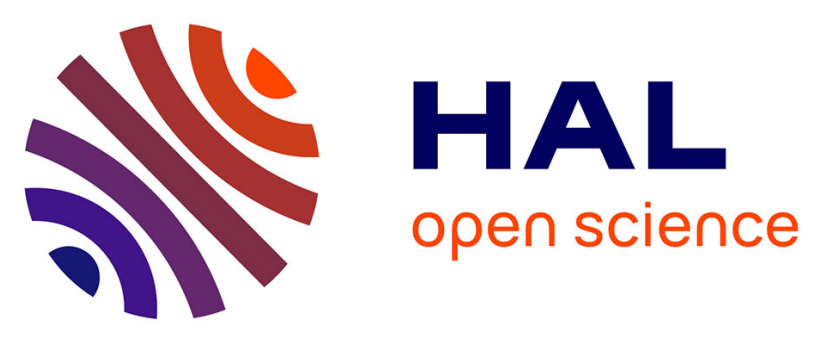

\title{
Coût de la radiothérapie des métastases osseuses en France: étude rétrospective monocentrique
}

\author{
C. Le Fèvre, I. Menoux, Y. Pin, D. Antoni, A. Thiéry, G. Noël
}

\section{To cite this version:}

C. Le Fèvre, I. Menoux, Y. Pin, D. Antoni, A. Thiéry, et al.. Coût de la radiothérapie des métastases osseuses en France: étude rétrospective monocentrique. Cancer/Radiothérapie, 2019, 23, pp.1 - 9. 10.1016/j.canrad.2018.01.009 . hal-03486456

\section{HAL Id: hal-03486456 https://hal.science/hal-03486456}

Submitted on 20 Dec 2021

HAL is a multi-disciplinary open access archive for the deposit and dissemination of scientific research documents, whether they are published or not. The documents may come from teaching and research institutions in France or abroad, or from public or private research centers.
L'archive ouverte pluridisciplinaire HAL, est destinée au dépôt et à la diffusion de documents scientifiques de niveau recherche, publiés ou non, émanant des établissements d'enseignement et de recherche français ou étrangers, des laboratoires publics ou privés.

\section{다)(1) $(5$}

Distributed under a Creative Commons Attribution - NonCommerciall 4.0 International 


\title{
Coût de la radiothérapie des métastases osseuses en France : étude rétrospective monocentrique
}

\section{Cost of radiotherapy for bone metastases in France: a monocentric retrospective study}

\author{
C. Le Fèvre ${ }^{1}$, I. Menoux ${ }^{1}$, Y. Pin ${ }^{1}$, D. Antoni ${ }^{1,2}$, A. Thiéry ${ }^{3}$, G. Noël ${ }^{1,2, *}$
}

${ }^{1}$ Département universitaire de radiothérapie, centre Paul-Strauss, Unicancer, 3, rue de la Porte-de1’Hôpital, 67065 Strasbourg cedex, France

${ }^{2}$ Laboratoire EA 3430, Fédération de médecine translationnelle de Strasbourg (FMTS), université de Strasbourg, 67000 Strasbourg, France

${ }^{3}$ Département de santé publique, centre Paul-Strauss, Unicancer, 3, rue de la Porte-de-1'Hôpital, 67065 Strasbourg cedex, France

* Auteur correspondant : G. Noël, MD, PhD ; adresse e-mail : gnoel@strasbourg.unicancer.fr

\section{Résumé}

Introduction : Le coût de la radiothérapie est une préoccupation pour les systèmes de santé. Les schémas classiques non fractionnés ou multifractionnés ont montré la même efficacité en termes de soulagement des douleurs mais un traitement non fractionné semble moins coûteux. Cependant, en pratique courante, les traitements multifractionnés restent encore majoritaires ce qui représente un surcoût pour la société. Par ailleurs, l'utilisation de la stéréotaxie se démocratise et offre de nouvelles perspectives curatives, mais à quel prix ?

Matériel et méthodes : Une étude rétrospective monocentrique réalisée au sein d'un département de radiothérapie français a été menée afin d'évaluer et de comparer le coût des irradiations des métastases osseuses non compliquées en fonction du schéma de radiothérapie choisi : 30 Gy en dix fractions, 20 Gy en cinq fractions, 8 Gy en une séance ou en conditions stéréotaxiques. 
Résultats : Entre janvier 2014 et décembre 2015, 91 patients ayant reçu 116 traitements ont été inclus dans l'étude, dont 44 hommes (48\%) et 47 femmes (52\%), âgés de 63 ans (25-88 ans). Trente-quatre traitements (29\%) étaient 30 Gy en dix fractions (groupe $30 \mathrm{~Gy}$ ), 24 (21\%) de 20 Gy en cinq fractions (groupe 20 Gy), 25 (22\%) par 8 Gy en séance fraction (groupe 8 Gy) et 33 traitements (28\%) en conditions stéréotaxiques (groupe stéréotaxie). Le coût d'une radiothérapie par stéréotaxie était significativement plus important que celui d'un traitement 3D classique ( $\mathrm{p}<0,001)$. Si l'on ajoutait à ce coût le coût du transport, la stéréotaxie restait la plus couteuse ( $\mathrm{p}<0,001)$. Le coût d'un traitement par 30 Gy était significativement plus important que celui d'un traitement par $20 \mathrm{~Gy}(\mathrm{p}=0,006)$ ou 8 Gy $(\mathrm{p}<0,001)$, cela même après avoir additionné le coût du transport $(\mathrm{p}<0,001)$, sans différence significative entre 20 Gy et 8 Gy $(p=0,11)$. En ce qui concerne le coût global du traitement, incluant le coût total des traitements, du transport associé et des ré-irradiations, la stéréotaxie était le traitement le plus coûteux $(\mathrm{p}<0,001)$. Le coût global était significativement plus important dans le groupe $30 \mathrm{~Gy}$ que dans celui Gy ( $\mathrm{p}=0,012)$ ou $8 \mathrm{~Gy}(\mathrm{p}=0,001)$, sans différence significative entre 20 Gy et 8 Gy $(\mathrm{p}=0,38)$. Il n'existait pas de différence significative du coût du suivi entre les groupes $30 \mathrm{~Gy}, 20 \mathrm{~Gy}, 8$ Gy et stéréotaxie à un mois $(\mathrm{p}=0,09)$ mais à trois mois $(\mathrm{p}=0,01)$ et six mois $(\mathrm{p}=0,0001)$ il était significativement plus important après un traitement tridimensionnel. Il n'y avait pas de différence significative du coût global incluant la radiothérapie initiale, le transport et l'ensemble du suivi sur six mois entre les groupes $(\mathrm{p}=0,04)$.

Conclusion : La stéréotaxie est une technique d'irradiation efficace et curative mais plus onéreuse.

Elle est à privilégier chez certains patients, ayant une espérance de vie plus longue, dans un contexte de traitement non palliatif. Le traitement non fractionné de 8 Gy paraît être le plus avantageux financièrement tout en concevant une efficacité équivalente aux traitements multifractionnés et en préservant la qualité de vie des patients.

\title{
Mots clés
}

Coûts ; Métastases osseuses ; Radiothérapie ; Soins palliatifs ; Stéréotaxie

\begin{abstract}
Purpose: The cost of radiotherapy is a concern for health systems. The conventional non fractionated or multifractionated schemes have shown the same efficacy in terms of pain relief but a non fractionated treatment seems less expensive. However, in general practice, multifractionated treatments are still the majority, which represents an additional cost for society. Moreover, the use of stereotactic body radiotherapy becomes more democratic and offers new curative perspectives, but at what price?
\end{abstract}

Material and methods: A monocentric retrospective study was conducted in a French radiotherapy department to evaluate and compare the cost of irradiation of uncomplicated bone metastases 
according to the selected radiotherapy regimen: $30 \mathrm{~Gy}$ in 10 fractions, $20 \mathrm{~Gy}$ in five fractions, $8 \mathrm{~Gy}$ in one fraction or stereotactic body radiotherapy.

Results: Between January 2014 and December 2015, 91 patients receiving 116 treatments were included in the study, including 44 men (48\%) and 47 women (52\%) were 63 years old (25-88 years). Thirty-four treatments (29\%) were performed by 30 Gy in 10 fractions (30 Gy group), 24 treatments (21\%) by $20 \mathrm{~Gy}$ in 5 fractions ( 20 Gy group), 25 treatments (22\%) by $8 \mathrm{~Gy}$ in one fraction ( $8 \mathrm{~Gy}$ group) and 33 treatments (28\%) by stereotactic body radiotherapy (SBRT group). The cost of stereotactic body radiotherapy was significantly higher than that of three-dimensional treatments $(\mathrm{p}<0.001)$. If the cost of transport was added to this cost, stereotactic body radiotherapy remained the most expensive $(\mathrm{p}<0.001)$. The cost of $30 \mathrm{~Gy}$ treatment was significantly higher than the cost of treatment with $20 \mathrm{~Gy}(\mathrm{p}=0.006)$ or $8 \mathrm{~Gy}(\mathrm{p}<0.001)$, even after adding the transport cost $(\mathrm{p}<0.001)$, with no significant difference between $20 \mathrm{~Gy}$ and $8 \mathrm{~Gy}(\mathrm{p}=0,11)$. For the overall cost of treatment including the total cost of treatments, associated transport and reirradiation, stereotactic body radiotherapy was the most expensive treatment $(\mathrm{p}<0.001)$ and this cost was significantly higher in the 30 Gy group than in the 20 Gy group ( $p=0.012$ ) or 8 Gy group ( $p=0.001$ ), with no significant difference between $20 \mathrm{~Gy}$ and $8 \mathrm{~Gy}(\mathrm{p}=0.38)$. There was no significant difference in the cost of follow-up between $30 \mathrm{~Gy}, 20 \mathrm{~Gy}, 8 \mathrm{~Gy}$ and stereotactic body radiotherapy at one month $(\mathrm{p}=0.09)$ but at three months $(\mathrm{p}=0.01)$ and six months $(\mathrm{p}=0.0001)$, this cost was significantly higher after a threedimensional treatment. There was no significant difference in overall cost including initial radiotherapy, transport and overall follow-up over 6 months between groups $(\mathrm{p}=0.04)$.

Conclusion : Stereotactic body radiotherapy is an efficient and curative irradiation technique but more expensive. It is preferred for some patients with a longer life expectancy in a non-palliative treatment setting. The $8 \mathrm{~Gy}$ treatment appears to be the most cost-effective while designing an equivalent efficiency to multifractionated treatments and preserving the quality of life of patients.

\section{Keywords}

Costs; Bone metastases; Radiotherapy; Palliative care; SBRT

\section{Introduction}

Les métastases osseuses représentent une évolution commune à de nombreux cancers. Environ deux tiers des patients atteints d'un cancer sont susceptibles de voir se développer des métastases osseuses au cours de l'évolution de la maladie [1]. Les métastases osseuses se localisent le plus 
souvent au niveau du rachis notamment thoracique et lombaire [2]. Elles peuvent causer des complications telles que douleurs, compressions, fractures, hypercalcémie et altèrent la qualité de vie dans $70 \%$ des cas [3].

Environ $50 \%$ des patients atteints d'un cancer bénéficieront d'une radiothérapie au cours de l'évolution de leur maladie [4], dont environ $8 \%$ au cours des 30 derniers jours de vie [5]. La radiothérapie a une place prépondérante dans la prise en charge globale des métastases osseuses et a pour objectif de soulager les douleurs, de limiter les complications et d'amélioration la qualité de vie [6]. Selon les études et d'après les définitions de Chow et al., les taux de réponse globale sur la diminution de la douleur sont compris entre $60 \%$ et $80 \%$ et les taux de réponse complète entre $25 \%$ et $30 \%$, quelle que soit l'intensité de la douleur initiale [7,8]. Les trois schémas d'irradiation tridimensionnelle, dits classiques, utilisés les plus fréquemment au niveau international sont 30 Gy en dix fractions, 20 Gy en cinq fractions et 8 Gy en une séance [9], même si plus de 100 schémas d'irradiation différents ont été recensés dans la littérature [10]. Il a été montré la non infériorité en termes de soulagement des douleurs des schémas classiques non-fractionnés par rapport à ceux fractionnés [11]. Par ailleurs, la radiothérapie en conditions stéréotaxiques se démocratise pour le traitement des métastases osseuses et offre de nouvelles perspectives au traitement puisque ce dernier devient potentiellement curateur.

En France, le coût d'une radiothérapie est calculé en fonction du nombre de séances réalisées et de la technique utilisée. De nombreuses études ont montré qu'un traitement non fractionné est moins coûteux qu'un traitement fractionné ; il paraitrait donc judicieux, pour des soucis d'économie de santé, d'utiliser un traitement non fractionné. Cependant, même si les organismes et sociétés savantes internationales recommandent l'utilisation d'un traitement non fractionné pour des métastases osseuses non compliquées, il existe une réticence mondiale à cette pratique, ce qui représente un surcoût sociétal.

Cette étude avait pour objectif d'évaluer les coûts engendrés pour la prise en charge des métastases osseuses non compliquées à partir de l'étude des pratiques courantes d'un service de radiothérapie français.

\section{Matériel et méthodes}

\subsection{Description des patients}

L'étude s'est déroulée au sein d'un département de radiothérapie français. Entre janvier 2014 et décembre 2015, les dossiers des patients traités pour des métastases osseuses ont été extraits par les codes Z51.01 pour « séance de radiothérapie » et C79.5 pour « tumeur maligne secondaire des os et de la moelle osseuse » à partir du logiciel Mosaiq ${ }^{\circledR}$ (Impac Medical Systems, Sunnyvale, États-Unis). 
Parmi ces derniers, les patients sélectionnés pour inclusion dans l'étude 1/ avaient plus de 18 ans, 2/ un cancer histologiquement prouvé, métastatique osseux, quel que soit le primitif, 3/ devaient être suivis exclusivement dans le centre où ils avaient reçu la radiothérapie, 4/ sans irradiation antérieure du site osseux concerné par l'étude, 5/ sans infiltration des tissus mous, 6/ sans compression médullaire ou fracture. Les sites irradiés correspondaient au volume cible planifié dans son intégralité. Si ce dernier comprenait plusieurs vertèbres adjacentes, un seul site était considéré. Sur la période d'étude, 420 dossiers de radiothérapie de métastases osseuses ont été identifiés et les dossiers de 116 traitements effectués chez 91 patients répondaient aux critères d'inclusion. L'ensemble des caractéristiques des patients et des traitements est résumé dans le tableau 1. La totalité des patients inclus présentait une maladie métastatique osseuse, survenue 23,7 mois en médiane après le diagnostic du cancer primitif. Soixante-quatorze patients avaient au moins deux localisations métastatiques osseuses et 56\% plus de cinq sites métastatiques osseux. Trente-deux patients (soit 35\%) étaient atteints d'oligométastases osseuses (au plus trois métastases osseuses), dont 17 avaient une seule (soit 19\%). Le diagnostic d'oligométastases osseuses était posé grâce aux examens complémentaires d'imagerie variables d'un patient à l'autre (TEP-scanographie, IRM). Par ailleurs, 53 patients inclus dans l'étude étaient atteints de métastases viscérales (soit 58\%), dont 34\% pulmonaires, 32\% ganglionnaires, $29 \%$ hépatiques et $10 \%$ cérébrales. Les sites osseux irradiés étaient le rachis et le sacrum pour 66 traitements (soit 57\%), le bassin pour 19 traitements (soit 16\%) et d'autres localisations pour 31 traitements (soit 27\%). Parmi les irradiations rachidiennes et sacrées, 28 concernaient le rachis dorsal seul, 12 le rachis lombaire seul, 12 le rachis dorsolombaire, sept le sacrum seul, cinq le rachis lombosacré et deux le rachis cervical seul. Sur les 91 patients inclus, 55 sont décédés (soit 60\%) dans un délai médian de 160 jours (extrêmes : 1-608 j) après la fin de la radiothérapie. La durée médiane de survie globale des patients était de 234 jours, 196 jours et 206 jours respectivement pour les groupes $30 \mathrm{~Gy}, 20$ Gy et 8 Gy. La durée médiane de survie n'était pas atteinte dans le groupe stéréotaxie.

\subsection{Traitements délivrés}

Les patients inclus ont bénéficié soit d'une irradiation conformationnelle tridimensionnelle de 30 Gy en dix fractions, 20 Gy en cinq fractions ou 8 Gy en une séance effectuée par des accélérateurs linéaires Primus ${ }^{\circledR}$ (Siemens ${ }^{\circledR}$, Münich, Allemagne, énergies X 6, 25 MV) ou Clinac ${ }^{\circledR}$ (Varian ${ }^{\circledR}$, Palo Alto, Etats-Unis, énergie X 6,15 MV), soit d'une irradiation en conditions stéréotaxiques délivrant 23,31 Gy en trois fractions réalisée sous NovalisTX ${ }^{\circledR}$ (Varian Medical Systems ${ }^{\circledR}$, Palo Alto, CA, ÉtatsUnis, énergie X $15 \mathrm{MV}$ ). Un traitement conformationnel avec modulation d'intensité réalisé sur Tomothérapie $^{\circledR}$ (Accuray ${ }^{\circledR}$, Madison, WI, Etats-Unis, énergie X $6 \mathrm{MV}$ ) a été nécessaire pour deux traitements. Les patients ont été classés dans le groupe 30 Gy, 20 Gy, 8 Gy ou stéréotaxie selon le schéma thérapeutique reçu. En cas de traitement concomitant de plusieurs métastases osseuses, le groupe attribué dépendait du traitement le plus long. Vingt-trois patients ont bénéficié de la 
radiothérapie concomitante de deux à trois localisations osseuses (soit 25\%), pour un total de 116 traitements. Le nombre de patients dans le groupe 30 Gy, 20 Gy, 8 Gy ou stéréotaxie ayant bénéficié du traitement concomitant de deux localisations métastatiques osseuses était respectivement de six, cinq, trois et six. Aucun patients des groupes 20 Gy et 8 Gy n'ont bénéficié de l'irradiation concomitante de trois localisations mais deux patients du groupe $30 \mathrm{~Gy}$ et un patient du groupe stéréotaxie en ont bénéficié. Pour six patients, le schéma d'irradiation délivré était différent d'une localisation à l'autre. Parmi l'ensemble des traitements, 34 étaient de $30 \mathrm{~Gy}$ en dix fractions (groupe $30 \mathrm{~Gy}$, soit 29\%), 24 de $20 \mathrm{~Gy}$ en cinq fractions (groupe $20 \mathrm{~Gy}$, soit 21\%), 25 de 8 Gy en une fraction (groupe 8 Gy, soit 22\%) et 33 en conditions stéréotaxiques (groupe stéréotaxie, soit 28\%).

\subsection{Calcul du cô̂t du traitement}

Les coûts entrepris pour le traitement par radiothérapie et l'ensemble du suivi des patients à la suite de ce traitement ont été collectés. Pour l'analyse, la perspective adoptée était celle du payeur identifié comme l'assurance maladie. L'horizon temporel était égal à six mois. Les coûts médicaux directs étaient représentés par la radiothérapie et les différents éléments du suivi du patient. Les coûts non médicaux indirects étaient représentés par le transport du patient. Le calcul des coûts a été évalué en fonction des données de la caisse primaire d'assurance maladie (CPAM) et du programme de médicalisation des systèmes d'information (PMSI).

Les coûts exclusivement engendrés par l'irradiation dépendaient du fractionnement (selon le nombre de séances effectuées), de la machine et de la technique utilisée (Annexe 1). L'ensemble des patients avait bénéficié d'une consultation auprès de l'oncologue radiothérapeute avant le début du traitement. Son coût n'était pas inclus dans la comparaison des coûts des irradiations car identique pour chaque patient. Le coût de la scanographie dosimétrique a été inclus dans une « séance de préparation » et avait été pris en compte en plus du coût des séances d'irradiation. Le coût de l'IRM dosimétrique requise pour la réalisation d'un traitement stéréotaxique a également été inclus dans celui de la « séance de préparation ». Le coût de la « séance de préparation » dépendait de la technique d'irradiation. Les consultations en cours de traitement ainsi que les imageries de contrôle au cours du traitement ont été incluses dans le coût de l'irradiation. Lors du traitement de deux ou trois localisations métastatiques osseuses concomitantes, le coût des séances était équivalent à celui de l'irradiation d'une seule localisation et dépendait du schéma le plus long. En revanche,, le coût de la séance de préparation était doublé ou triplé selon le nombre de localisations irradiées. Les coûts en lien avec le transport sont présentés en Annexes 2, 3, 4, 5 et 6. Le coût du suivi, incluant les hospitalisations et consultations après la radiothérapie, la prise en charge des complications et des réirradiations, a été recueilli grâce au logiciel $\mathrm{GiHM}^{\circledR}$ utilisé dans le centre de l'étude. 


\subsection{Statistiques}

Les données concernant le sexe, l'âge, le cancer primitif, l'histoire thérapeutique et métastatique, le traitement de radiothérapie, l'évolution de la maladie et les coûts engendrés par le traitement ont été recueillis jusqu'au 3 juin 2016. Les cas de réirradiations, de compression médullaire, de fracture pathologique et la toxicité aiguë immédiate du traitement basée sur les grades de la Common Terminology Criteria for Adverse Events, version 4.0 (CTCAE v4) ont été recherchés. Le nombre de kilomètres parcourus par le patient jusqu'au centre a été obtenu grâce au site Viamichelin ${ }^{\circledR}$ (https://www.viamichelin.fr). Le mode de transport utilisé par le patient a été recueilli (ambulance, véhicule sanitaire léger, voiture particulière, transport en commun ou autre).

Pour l'analyse des coûts engendrés par le traitement, les patients ont été classés dans le groupe 30 Gy, 20 Gy, 8 Gy ou stéréotaxie selon le schéma thérapeutique reçu.

L'analyse statistique comprenait une partie descriptive et une partie inférentielle. L'analyse statistique descriptive des variables quantitatives a été réalisée en donnant pour chaque variable les paramètres de position (moyenne, médiane, minimum, maximum, premier et troisième quartiles) ainsi que les paramètres de dispersion (variance, écart type, étendue et écart interquartile). Le caractère gaussien des données était vérifié graphiquement et testé par le test de Shapiro Wilk. L'analyse statistique descriptive des variables qualitatives a été effectuée en donnant les effectifs et les proportions de chaque modalité dans l'échantillon. Pour les variables quantitatives, les modalités de traitement ont été comparées par une analyse de variance lorsque la distribution était normale ou par le test de Kruskal et Wallis dans le cas contraire. Pour les variables qualitatives, les modalités ont été comparées par test de Chi 2 lorsque les effectifs théoriques étaient suffisants ou par le test exact de Fisher dans le cas contraire.

\section{Résultats}

\subsection{Coûts globaux des traitements}

Le coût total de l'ensemble des traitements associés aux transports était de $298705 €$ et le coût moyen par patient était de $3282 €$ (intervalle : 135-7781 €). Le coût moyen par patient en fonction du schéma thérapeutique était respectivement de $3291 €$ (intervalle : 952-7225€), $1839 €$ (extrêmes : 272$3456 €$ ), $1151 €$ (intervalle : $135-2373 €$ ) et $5668 €$ (intervalle : 4 143-7781€) dans les groupes $30 \mathrm{~Gy}$, 20 Gy, 8 Gy et stéréotaxie ( $p<0,001)$. Le coût global du traitement incluant le coût total des irradiations, du transport associé et des réirradiations était de $307708 €$. Dans les groupes $30 \mathrm{~Gy}, 20$ Gy, 8 Gy et stéréotaxie, il était respectivement de $95450 €, 39433 €, 21989 €$ et $150835 €$. Le coût moyen par patient selon les groupes était respectivement de $3291 €$ (intervalle : 952-7225€), $1878 €$ (intervalle : $272-4131 €$ ), $1466 €$ (intervalle : $135-3634 €$ ) et $5801 €$ (intervalle : 4319-8676€) ( $\mathrm{p}<0,001)$. Le coût global incluant la radiothérapie initiale, le transport et l'ensemble du suivi sur six 
mois était de $965664 €$. Dans les groupes 30 Gy, 20 Gy, 8 Gy et stéréotaxie, il était respectivement de $333533 €, 288724 €, 128434 €$ et $214972 €$. Le coût moyen par patient était respectivement de 11 $501 €$ (intervalle : $2850-20870 €$ ), $13749 €$ (intervalle : 272-34 $136 €$ ), $8562 €$ (intervalle : 2336-20 $998 €)$ et $8268 €$ (intervalle : 5276-22 $457 €)(\mathrm{p}=0,04)$. Les coûts relatifs au traitement et au suivi des patients sont présentés tableau 2.

\subsection{Coût de l'irradiation initiale seule}

Le coût total des traitements, tout schéma confondu, s'élevait à $262622 €$ et le coût moyen du traitement par patient était de $2886 €$ (intervalle : 135-7380€). En fonction des groupes $30 \mathrm{~Gy}, 20 \mathrm{~Gy}$, 8 Gy et stéréotaxie, le coût total des irradiations était respectivement de $72984 €, 32346 €, 16096 €$ et $141196 €(\mathrm{p}<0,001)$. Le coût moyen de l'irradiation, dans les groupes $30 \mathrm{~Gy}, 20 \mathrm{~Gy}, 8 \mathrm{~Gy}$ et stéréotaxie, était respectivement de $2517 €$ (intervalle : 952-5556€), $1540 €$ (intervalle : 272-2676€), $1071 €$ (intervalle : $135-2167 €)$ et $5430 €$ (intervalle : 4116-7380€) $(\mathrm{p}<0,001)$.

\subsection{Coût des transports}

La distance médiane du logement du patient au centre était de $35 \mathrm{~km}$ (intervalle : 1-327 km). Le lieu d'habitation était localisé dans le département du centre de radiothérapie dans $68 \%$ des cas et dans un département limitrophe dans $32 \%$ des cas. La distance totale médiane parcourue pour l'ensemble du traitement par patient était de $132 \mathrm{~km}$ (intervalle : 0-2340 km) et le nombre médian de trajets aller ou retour réalisés pris individuellement était de six (intervalle : 0-20). Le transport utilisé pour se rendre aux séances de radiothérapie était le véhicule sanitaire léger dans $57 \%$ des cas, le transport interne à l'hôpital dans $18 \%$ des cas, le véhicule personnel dans $12 \%$ des cas, les transports en commun dans $6 \%$ des cas, l'ambulance dans $4 \%$ des cas et un autre mode dans 3\% des cas. Le coût total du transport de l'ensemble des traitements était de $36083 €$ et son coût moyen par traitement et par patient de 397 $€$ (intervalle : 0-2281 €). Dans les groupes 30 Gy, 20 Gy, 8 Gy et stéréotaxie, le coût total du transport était respectivement de $22466 €, 6277 €, 1175 €$ et $6164 €$ et le coût moyen de $775 €$ (intervalle : 0$2281 €$ ), $299 €$ (intervalle : 0-1603 $€$ ), $78 €$ (intervalle : $0-257 €$ ) et $237 €$ (intervalle : 0-743 €) $(\mathrm{p}=0,004)$.

\subsection{Coût post-thérapeutique}

Neuf sites ont été réirradiées dans un délai de 13 à 434 jours après le premier traitement (soit 8\%) et 17 patients ont bénéficié de l'irradiation d'une nouvelle localisation métastatique osseuse. Le coût total des neuf réirradiations s'élevait à $9003 €$. Dans les groupes 20 Gy, 8 Gy et stéréotaxie, il était respectivement de $810 €$ pour deux réirradiations (l'une par $20 \mathrm{~Gy}$ en cinq fractions, l'autre par 8 Gy en une fraction), $4718 €$ pour cinq réirradiations (dont une par $30 \mathrm{~Gy}$ en dix fractions, une par $20 \mathrm{~Gy}$ en cinq fractions, une par 13 Gy en deux fractions et deux par 8 Gy en une séances) et de $3475 €$ pour 
deux réirradiations (l'une par 30 Gy en dix fractions, l'autre par 8 Gy en une fraction) ( $p=0,26)$. Aucune réirradiation n'avait été effectué dans le groupe 30 Gy.

Le coût total de l'irradiations des nouvelles localisations métastatiques osseuses diagnostiquées au cours du suivi était de $7472 €, 18711 €, 22779 €$ et $49090 €$ respectivement à 1 mois, 3 mois, 6 mois et plus de 6 mois après la première irradiation. Il n'existait pas de différence significative du coût de l'irradiation des nouvelles localisations osseuses entre les groupes 30 Gy, 20 Gy, 8 Gy et stéréotaxie à 1 mois $(\mathrm{p}=0,29), 3$ mois $(\mathrm{p}=0,24), 6$ mois $(\mathrm{p}=0,97)$ et au-delà de 6 mois après la première irradiation $(\mathrm{p}=0,75)$. Le coût total du suivi, incluant les consultations et hospitalisations, le coût des ré-irradiations et le coût de l'irradiation de nouvelles localisations métastatiques osseuses, à 1 mois, 3 mois et 6 mois était de $259732 €, 436403 €$ et $666959 €$. Il n'existait pas de différence significative du coût du suivi entre les groupes $30 \mathrm{~Gy}, 20 \mathrm{~Gy}, 8 \mathrm{~Gy}$ et stéréotaxie à 1 mois $(\mathrm{p}=0,09)$ mais à 3 mois $(\mathrm{p}=0,01)$ et 6 mois $(\mathrm{p}=0,0001)$ une différence significative existait.

\subsection{Analyse comparative du coût des trois modalités tridimensionnelles}

En analysant uniquement les trois modalités de radiothérapie tridimensionnelles, le coût du traitement de 30 Gy était significativement plus important que le coût du traitement de $20 \mathrm{~Gy}(\mathrm{p}=0,006)$ ou 8 Gy $(\mathrm{p}<0,001)$, sans différence significative entre $20 \mathrm{~Gy}$ et $8 \mathrm{~Gy}(\mathrm{p}=0,11)$. Le coût du transport associé à la radiothérapie était également significativement plus important pour un traitement de 30 Gy que pour de 20 Gy ( $p=0,019)$ ou 8 Gy ( $p=0,005)$ sans différence significative entre 20 Gy et 8 Gy ( $p=0,07)$. En conséquence, le coût comprenant la radiothérapie et le transport était significativement plus important

après un traitement de $30 \mathrm{~Gy}$ que de $20 \mathrm{~Gy}(\mathrm{p}=0,0009)$ et $8 \mathrm{~Gy}(\mathrm{p}<0,001)$, sans différence significative entre 20 Gy et $8 \mathrm{~Gy}(\mathrm{p}=0,07)$. Le coût global du traitement incluant la radiothérapie initiale, le transport et les ré-irradiations était significativement plus important dans le groupe 30 Gy que 20 Gy $(\mathrm{p}=0,012)$ ou $8 \mathrm{~Gy}(\mathrm{p}=0,001)$, sans différence significative entre 20 Gy et $8 \mathrm{~Gy}(\mathrm{p}=0,38)$. Le coût du suivi ainsi que les coûts engagés pour l'irradiation d'autres localisations métastatiques osseuses au cours du suivi ne différaient pas significativement entre les groupes 30 Gy, 20 Gy et 8 Gy. Le coût global de la prise en charge incluant l'ensemble des irradiations et du suivi ne différait pas de manière significative entre les groupes $30 \mathrm{~Gy}, 20 \mathrm{~Gy}$ et $8 \mathrm{~Gy}(\mathrm{p}=0,24)$.

\section{Discussion}

Le coût de la radiothérapie palliative des métastases osseuses non compliquées est à prendre en compte dans les dépenses de santé liées à la prise en charge de la pathologie cancéreuse [12]. Il reste néanmoins à mettre en balance face aux coûts engendrés par l'ensemble des autres traitements des cancers métastatiques. Dans une étude prospective française, le coût de la première année de prise en charge des métastases osseuses de cancers pulmonaires était en moyenne $3999 €$ (intervalle de confiance à 95\% : 374-15 886) [13]. Une étude belge menée par Van de Werf et al. a montré qu'entre 2000 et 2009 le coût moyen d'une irradiation de 8 Gy en une séance, 20 Gy en cinq fractions et 30 Gy 
en dix fractions avait respectivement augmenté de $20 \%, 18 \%$ et 36\% [14]. La radiothérapie semble avoir un coût inférieur à celui de certains autres traitements symptomatiques des métastases osseuses. Konski et al. ont montré qu'une irradiation non fractionnée était moins coûteuse qu'une irradiation fractionnée, une chimiothérapie ou un traitement morphinique [15]. Macklis et al. ont exposé que le coût de la prise en charge des métastases osseuses sur 9 mois incluant une radiothérapie était de 1200 à $2500 \$$ alors qu'il était de 9000 à 36000 \$ pour une prise en charge par morphiniques seule [16].

Notre étude a montré que le coût de la radiothérapie palliative tridimensionnelle des métastases osseuses non compliquées pour le système de santé était significativement plus important après un traitement de 30 Gy qu'après 20 Gy ou 8 Gy. Cette différence existait également si l'on additionnait les coûts d'éventuelles ré-irradiations même si le taux de réirradiation était significativement plus important après un traitement de $8 \mathrm{~Gy}(\mathrm{p}=0,02)$. En outre, les coûts liés au transport était également significativement plus important pour un traitement de 30 Gy que de 20 Gy ou 8 Gy. Le coût de la radiothérapie non fractionnée était respectivement inférieur de $57 \%$ et $30 \%$ à celui du traitement fractionné de 30 Gy ou 20 Gy. Le coût global du traitement non fractionné, incluant la radiothérapie initiale, le transport et l'ensemble du suivi sur six mois, était respectivement de $54 \%$ et $52 \%$ inférieur au coût du traitement fractionné de 30 Gy ou 20 Gy. Néanmoins, au regard du coût global du traitement palliatif et du suivi du patient sur 6 mois, il n'y avait pas de différence significative entre les groupes recevant $30 \mathrm{~Gy}, 20 \mathrm{~Gy}$ ou $8 \mathrm{~Gy}$. Cependant, les différents groupes de patients de l'étude n'étaient pas homogènes et non directement comparables car l'étude était rétrospective et que le profil des patients inclus dans les différents groupes variait. Les caractéristiques des patients bénéficiant d'une stéréotaxie différaient de celles des patients recevant un traitement tridimensionnel, notamment l'état général, le stade et l'évolution de la maladie, les traitements anticancéreux antérieurs, ou encore les perspectives curatives. En outre, le traitement non fractionné était très minoritaire.

Plusieurs auteurs se sont intéressés au coût de la radiothérapie des métastases osseuses selon le fractionnement utilisé. Pour certains d'entre eux, le traitement non fractionné paraissait le plus avantageux. L'analyse économique de l'étude du Dutch Bone Metastasis, qui comparait un traitement de 8 Gy en une séance contre 24 Gy en six fractions, a montré que le coût de la radiothérapie non fractionnée était de $25 \%$ inférieur à celui de la radiothérapie fractionnée. Cette différence se réduisait à $7 \%$ quand le coût des réirradiations était pris en compte, les réirradiations étant significativement plus fréquentes dans le groupe recevant 8 Gy que dans celui recevant 24 Gy [17]. En outre, le coût global moyen d'un traitement non fractionné, incluant le transport, les hospitalisations ou encore les aides à domicile était également significativement inférieur à celui d'un traitement fractionné ( $p<0,001)$. Il en était de même si les coûts étaient corrélés avec la qualité de vie et la survie $(\mathrm{p}<0,05)$ [17]. Dans l'analyse économique du Radiation Therapy Oncology Group (RTOG) 9714, une radiothérapie de 30 Gy en dix fractions semblait être plus avantageuse en termes de coût/efficacité qu'une par 8 Gy en une 
séance. Cela s'expliquait par le nombre de réirradiations significativement plus important après un traitement de 8 Gy. En outre, une différence de 2 mois de la Quality Adjusted Life Year (QALY) entre les deux groupes a été mise en évidence, pour un coût doublé, en faveur de la radiothérapie fractionnée. Cependant cette différence était minime et le ratio était de 6973 \$/QALY. Les auteurs ont donc conclu que d'un point de vue économique le traitement de 8 Gy était à favoriser [18]. Bekelman et al. ont analysé 3050 dossiers de patients atteints de métastases osseuses d'un cancer prostatique. Le coût de la radiothérapie non fractionnée était inférieur à celui d'une radiothérapie fractionnée, mais le coût global des traitements était équivalent $(\mathrm{p}=0,16)$ [19]. Une étude néozélandaise a récemment montré qu'un traitement non fractionné des métastases osseuses des cancers du sein, de la prostate et du poumon était préférable en termes de coût-efficacité-qualité de vie [20]. Bayazidi et al., quant à eux, ont conclu qu'en termes de coût/efficacité un traitement fractionné était à favoriser car il comportait moins de retraitement et retrouvait un ratio coût/utilité était de 428 \$/QALY. Le traitement non fractionné était cependant moins couteux [21]. Dans la plupart des études, la réirradiation est plus fréquente après un traitement non fractionné, ce qui augmente le coût global de ce traitement par comparaison à un traitement fractionné. Les recommandations pour effectuer une réirradiation sont l'absence de contrôle de la douleur ou la récidive de la douleur après l'obtention d'une réponse. Une réirradiation ne devrait pas être effectuée avant 4 semaines après la fin de la première irradiation puisque la réponse au traitement peut être différée [6,7]. Dans les essais, le choix d'une seconde irradiation est souvent laissé libre au praticien et reste subjectif. La réirradiation est effectuée plus précocement, pour des scores douloureux plus faibles et une consommation d'antalgiques plus faible après un traitement non fractionné qu'un traitement fractionné [22].

Plusieurs facteurs économiques peuvent influencer le choix du fractionnement. En effet, Lievens et al. ont suggéré que le mode de remboursement avait une influence sur le choix du fractionnement [23]. Entre 2000 et 2006, la Belgique avait modifié son mode de remboursement. Les traitements non fractionnés ayant été revalorisés, ils ont été plus souvent prescrits [24]. En France, les coûts pour le système de santé engendrés par la radiothérapie sont calculés selon le nombre de séances effectuées, la machine d'irradiation et la technique d'irradiation utilisées. Il semble donc qu'un traitement en plusieurs séances utilisant des techniques d'irradiation plus précises et des machines dédiées plus spécifiques soit plus rentable pour un service de radiothérapie mais plus couteux au système de santé. De nombreux essais randomisés ont prouvé que les traitements de 30 Gy, 20 Gy et 8 Gy avaient la même efficacité en termes de soulagement des douleurs. Les recommandations internationales sont l'utilisation du traitement non fractionné de 8 Gy pour des métastases osseuses non compliquées et cela même s'il expose le patient à un risque supérieur de réirradiation. Malgré cela, le traitement non fractionné reste sous-utilisé, comme l'ont montré Rutter et al. dans une étude rétrospective publiée en 2015, puisqu'entre 2005 et 2011 le taux de traitement non fractionné prescrit était passé de 3,4\% à 7,5\% aux Etats-Unis. Le schéma de 30 Gy en dix fractions a été prescrit chez 
57\% des patients inclus dans l'étude [25]. En outre, en utilisant un traitement de $30 \mathrm{~Gy}$, la qualité de vie du patient en fin de vie peut être altérée car le traitement l'oblige à passer davantage de temps à l'hôpital et dans les transports. En outre, l'augmentation de l'espérance de vie des patients expose à une augmentation du nombre de réirradiations et à l'irradiation de nouvelles localisations. Il semble donc important de mesurer la balance entre efficacité, confort du patient et coût lors du choix du fractionnement de radiothérapie [23]. Pour des soucis d'économie des dépenses de santé, il semble que la radiothérapie non fractionné soit le traitement le moins couteux des systèmes de santé, pour une efficacité similaire aux traitements fractionnés, et devrait donc être utiliser pour le traitement palliatif des métastases osseuses non compliquées.

Grâce à l'avancée des techniques d'irradiation, la stéréotaxie a pris sa place dans le traitement des métastases osseuses, notamment rachidiennes. Elle permet non seulement de soulager les douleurs liées à la métastase osseuse avec une efficacité qui semble être supérieure au traitement tridimensionnelle, mais également d'espérer une stérilisation du site métastatique en cas de cancer de pronostic favorable, avec un taux de contrôle local d'environ 90\% [26-29]. Elle offre donc une approche curative des lésions en délivrant une forte dose par fraction dans un volume réduit tout en protégeant les organes à risques [30-35]. La stéréotaxie semble être à favoriser dans certains cas comme les cas d'oligométastases osseuses ou de tumeurs considérées comme radiorésistantes [36]. Cependant, d'après les données de la littérature, son coût semble supérieur à celui des traitements tridimensionnels classiques. Dans l'étude de Haley et al. la stéréotaxie représentait un coût supérieur à la radiothérapie tridimensionnelle puisque pour 100 patients, ce dernier était de 842420 \$ pour un traitement stéréotaxique, de 676309 \$ pour une irradiation de 30 Gy en dix fractions et de 499911 \$ pour un traitement de 20 Gy en cinq fractions [37]. Dans l'étude de Amdur et al, la radiothérapique en conditions stéréotaxiques était comparée à une irradiation de 20 Gy en cinq fractions ou 30 Gy en dix fractions. Même en incluant les coûts engendrés par les réirradiations, le traitement tridimensionnel restait moins onéreux que celui en conditions stéréotaxiques. Un traitement de 30 Gy ou 20 Gy représentait $80 \%$ et $59 \%$ respectivement du coût d'un traitement en conditions stéréotaxiques [37]. Kim et al. ont analysés le coût/efficacité de la radiothérapie en conditions stéréotaxiques par comparaison à la radiothérapie externe classique selon le soulagement des douleurs, l'amélioration de la qualité de vie et l'augmentation de la survie. Il y avait une supériorité de la radiothérapie stéréotaxique en termes de soulagement des symptômes, avec un taux de contrôle des douleurs de $80 \%$ contre $60 \%$ avec un traitement tridimensionnel classique. Et pourtant, la stéréotaxie n'était pas rentable selon le rapport coût-QALY. En conclusion, la radiothérapie en conditions stéréotaxiques devait être indiquée chez des patients sélectionnés dont l'espérance de vie serait supérieure à 11 mois [38]. D'autres études ont montré une diminution des coûts indirects avec la technique stéréotaxique [39]. Notre étude a montré que le coût du traitement seul était significativement plus important pour un traitement stéréotaxique que pour un traitement tridimensionnel et cela même en additionnant le 
coût du transport associé. Même si le taux de ré-irradiation était significativement plus important après un traitement de $8 \mathrm{~Gy}(\mathrm{p}=0,02)$, le coût de la réirradiation ne différait pas significativement entre les groupes et le coût du traitement associé à celui des réirradiations restait plus élevé après un traitement stéréotaxique. En revanche, le coût total du suivi, incluant les réirradiations, les nouvelles irradiations, les consultations et les hospitalisations, variait significativement à 3 mois et 6 mois en faveur de la radiothérapie en conditions stéréotaxiques. Enfin, le coût global du traitement associé à l'ensemble du suivi variait significativement entre les radiothérapies stéréotaxiques et tridimensionnelles, en faveur de celle en conditions stéréotaxiques. Le traitement stéréotaxique pris isolément semble plus onéreux qu'un traitement tridimensionnel classique mais il peut être curatif en plus de son action symptomatique. Toutefois, ce traitement s'adresse à des patients sélectionnés qui ne présentent pas forcément de douleurs et qui ont une espérance de vie supérieure. Il est donc difficile de juger le bénéfice apporté par la technique stéréotaxique par comparaison à celle tridimensionnelle en termes de qualité de vie et de survie chez des patients non comparables. Le suivi n'a été évalué que sur les 6 mois suivant la fin de la radiothérapie mais l'on peut supposer que le suivi après radiothérapie stéréotaxique sera plus long et donc éventuellement plus couteux à long terme. Il ne semble de ce fait peut-être pas judicieux de comparer le coût de ces deux types de traitements et du suivi car ils ne s'adressent pas aux mêmes patients.

\section{Conclusion}

Le coût de la radiothérapie palliative des métastases osseuses non compliquées doit s'intégrer dans les dépenses de santé liées à la prise en charge de la pathologie cancéreuse. Afin de participer aux économies de santé, il semble important d'évaluer les coûts des traitements pour les systèmes de santé. Ainsi, le traitement non fractionné de 8 Gy paraît être le plus avantageux financièrement tout en concevant une efficacité équivalente aux traitements fractionnés et en préservant la qualité de vie des patients. La stéréotaxie est une technique d'irradiation à privilégier chez certains patients, ayant une espérance de vie plus longue, dans un contexte de traitement non palliatif.

\section{Conflits d'intérêts}

Les auteurs déclarent ne pas avoir de conflit d'intérêts.

\section{Annexe 1}

Coût d'une séance de radiothérapie en fonction de la machine utilisée (en euros).

Cost of a radiotherapy session according to the machine used (in euros). 


\section{Annexe 2}

Coûts du transport

Cost of transport

\section{Annexe 3}

Tarifs des transports sanitaires par ambulance (tarifs en euros applicables au $1^{\text {er }}$ février 2015)

Rates for medical transports by ambulance (tariffs in euros applicable on 1 February 2015)

\section{Annexe 4}

Liste des communes concernées par le forfait agglomération (tarifs conventionnels de l'Assurance maladie pour les ambulances).

List of French municipalities concerned by patient transport reimbursement plan.

\section{Annexe 5}

Tarifs des transports sanitaires par véhicule sanitaire léger (en euros ; applicables au 1er janvier 2015)

Rates for patient transport vehicle (in euros; applicable on 1 January 2015)

\section{Annexe 6}

Zones pour la tarification du forfait départemental des véhicules sanitaires légers.

Zone pricing in France for transport reimbursement plan of patient transport vehicle expenses.

\section{Bibliographie}

[1] Shaw B, Mansfield FL, Borges L. One-stage posterolateral decompression and stabilization for primary and metastatic vertebral tumors in the thoracic and lumbar spine. $\mathrm{J}$ Neurosurg 1989;70:405-10. doi:10.3171/jns.1989.70.3.0405.

[2] van Oorschot B, Rades D, Schulze W, Beckmann G, Feyer P. Palliative radiotherapy--new approaches. Semin Oncol 2011;38:443-9. doi:10.1053/j.seminoncol.2011.03.015.

[3] Papatheofanis FJ, Williams E, Chang SD. Cost-utility analysis of the cyberknife system for $\begin{array}{llll}\text { metastatic } & \text { spinal } & \text { tumors. } & \text { Neurosurgery }\end{array}$;4:A73-83. 
doi:10.1227/01.NEU.0000341205.37067.DE.

[4] Delaney G, Jacob S, Featherstone C, Barton M. The role of radiotherapy in cancer treatment: estimating optimal utilization from a review of evidence-based clinical guidelines. Cancer 2005;104:1129-37. doi:10.1002/cncr.21324.

[5] Guadagnolo BA, Liao K-P, Elting L, Giordano S, Buchholz TA, Shih Y-CT. Use of radiation therapy in the last 30 days of life among a large population-based cohort of elderly patients in the United States. J Clin Oncol 2013;31:80-7. doi:10.1200/JCO.2012.45.0585.

[6] Thureau S, Vieillard M-H, Supiot S, Lagrange J-L. Radiothérapie des métastases osseuses. Cancer Radiother 2016;20 Suppl:S227-34. doi:10.1016/j.canrad.2016.07.027.

[7] Chow E, Hoskin P, Mitera G, Zeng L, Lutz S, Roos D, et al. Update of the international consensus on palliative radiotherapy endpoints for future clinical trials in bone metastases. Int J Radiat Oncol 2012;82:1730-7. doi:10.1016/j.ijrobp.2011.02.008.

[8] Lutz S, Balboni T, Jones J, Lo S, Petit J, Rich SE, et al. Palliative radiation therapy for bone metastases: Update of an ASTRO Evidence-Based Guideline. Pract Radiat Oncol 2017;7:4-12. doi:10.1016/j.prro.2016.08.001.

[9] Fairchild A, Barnes E, Ghosh S, Ben-Josef E, Roos D, Hartsell W, et al. International patterns of practice in palliative radiotherapy for painful bone metastases: evidence-based practice? Int J Radiat Oncol 2009;75:1501-10. doi:10.1016/j.ijrobp.2008.12.084.

[10] Maher EJ, Coia L, Duncan G, Lawton PA. Treatment strategies in advanced and metastatic cancer: differences in attitude between the USA, Canada and Europe. Int J Radiat Oncol Biol Phys $1992 ; 23: 239-44$.

[11] Wu JS-Y, Wong R, Johnston M, Bezjak A, Whelan T, Cancer Care Ontario Practice Guidelines Initiative Supportive Care Group. Meta-analysis of dose-fractionation radiotherapy trials for the palliation of painful bone metastases. Int J Radiat Oncol Biol Phys 2003;55:594-605. 
[12] Barton MB, Jacob SA, Gebsky V. Utility-adjusted analysis of the cost of palliative radiotherapy for bone metastases. Australas Radiol 2003;47:274-8.

[13] Decroisette C, Monnet I, Berard H, Quere G, Le Caer H, Bota S, et al. Epidemiology and treatment costs of bone metastases from lung cancer: a French prospective, observational, multicenter study (GFPC 0601). J Thorac Oncol 2011;6:576-82. doi:10.1097/JTO.0b013e318206a1e3.

[14] Van de Werf E, Verstraete J, Lievens Y. The cost of radiotherapy in a decade of technology evolution. Radiother Oncol 2012;102:148-53. doi:10.1016/j.radonc.2011.07.033.

[15] Konski A. Radiotherapy is a cost-effective palliative treatment for patients with bone metastasis from prostate cancer. Int J Radiat Oncol Biol Phys 2004;60:1373-8. doi:10.1016/j.ijrobp.2004.05.053.

[16] Macklis RM, Cornelli H, Lasher J. Brief courses of palliative radiotherapy for metastatic bone pain: a pilot cost-minimization comparison with narcotic analgesics. Am J Clin Oncol 1998;21:61722.

[17] Hout WB van den, Linden YM van der, Steenland E, Wiggenraad RGJ, Kievit J, Haes H de, et al. Single- versus multiple-fraction radiotherapy in patients with painful bone metastases: cost-utility analysis based on a randomized trial. J Natl Cancer Inst 2003;95:222-9. doi:10.1093/jnci/95.3.222.

[18] Konski A, James J, Hartsell W, Leibenhaut MH, Janjan N, Curran W, et al. Economic Analysis of Radiation Therapy Oncology Group (RTOG) 97-14: Multiple versus single fraction radiation treatment of patients with bone metastases. Am J Clin Oncol 2009;32:423-8. doi:10.1097/COC.0b013e31818da9f7.

[19] Bekelman JE, Epstein AJ, Emanuel EJ. Single- vs multiple-fraction radiotherapy for bone metastases from prostate cancer. JAMA 2013;310:1501-2. doi:10.1001/jama.2013.277081.

[20] Collinson L, Kvizhinadze G, Nair N, McLeod M, Blakely T. Economic evaluation of singlefraction versus multiple-fraction palliative radiotherapy for painful bone metastases in breast, lung and prostate cancer. J Med Imaging Radiat Oncol 2016;60:650-60. doi:10.1111/1754-9485.12467. 
[21] Bayazidi Y, Keshtkaran A, Homaie Rad E, Ansari M, Javanbakht M, Hashemi Meshkini A, et al. Cost-utility analysis of single-fraction versus multiple-fraction radiotherapy in patients with painful bone metastases: an Iranian patient's perspective study. Value Health Reg Issues 2017;12:84-9. doi:10.1016/j.vhri.2016.10.004.

[22] van der Linden YM, Lok JJ, Steenland E, Martijn H, van Houwelingen H, Marijnen CAM, et al. Single fraction radiotherapy is efficacious: a further analysis of the Dutch Bone Metastasis Study controlling for the influence of retreatment. Int J Radiat Oncol Biol Phys 2004;59:528-37. doi:10.1016/j.jirobp.2003.10.006.

[23] Lievens Y, Van den Bogaert W, Rijnders A, Kutcher G, Kesteloot K. Palliative radiotherapy practice within Western European countries: impact of the radiotherapy financing system? Radiother Oncol J Eur Soc Ther Radiol Oncol 2000;56:289-95.

[24] van der Linden Y, Roos D, Lutz S, Fairchild A. International variations in radiotherapy fractionation for bone metastases: geographic borders define practice patterns? Clin Oncol 2009;21:655-8. doi:10.1016/j.clon.2009.08.004.

[25] Rutter CE, Yu JB, Wilson LD, Park HS. Assessment of national practice for palliative radiation therapy for bone metastases suggests marked underutilization of single-fraction regimens in the United States. Int J Radiat Oncol 2015;91:548-55. doi:10.1016/j.ijrobp.2014.10.045.

[26] Gerszten PC, Burton SA, Ozhasoglu C, Welch WC. Radiosurgery for spinal metastases: clinical experience in 500 cases from a single institution. Spine 2007;32:193-9. doi:10.1097/01.brs.0000251863.76595.a2.

[27] Bhattacharya IS, Hoskin PJ. Stereotactic body radiotherapy for spinal and bone metastases. Clin Oncol R Coll Radiol 2015;27:298-306. doi:10.1016/j.clon.2015.01.030.

[28] Amdur RJ, Bennett J, Olivier K, Wallace A, Morris CG, Liu C, et al. A prospective, phase II study demonstrating the potential value and limitation of radiosurgery for spine metastases. Am J Clin Oncol 2009;32:515-20. doi:10.1097/COC.0b013e318194f70f. 
[29] Hall WA, Stapleford LJ, Hadjipanayis CG, Curran WJ, Crocker I, Shu H-KG. Stereotactic body radiosurgery for spinal metastatic disease: an evidence-based review. Int $\mathrm{J}$ Surg Oncol 2011;2011:979214. doi:10.1155/2011/979214.

[30] De Bari B, Alongi F, Mortellaro G, Mazzola R, Schiappacasse L, Guckenberger M. Spinal metastases: Is stereotactic body radiation therapy supported by evidences? Crit Rev Oncol Hematol 2016;98:147-58. doi:10.1016/j.critrevonc.2015.11.002.

[31] Degen JW, Gagnon GJ, Voyadzis J-M, McRae DA, Lunsden M, Dieterich S, et al. CyberKnife stereotactic radiosurgical treatment of spinal tumors for pain control and quality of life. J Neurosurg Spine 2005;2:540-9. doi:10.3171/spi.2005.2.5.0540.

[32] Gerszten PC, Burton SA, Belani CP, Ramalingam S, Friedland DM, Ozhasoglu C, et al. Radiosurgery for the treatment of spinal lung metastases. Cancer 2006;107:2653-61. doi:10.1002/cncr.22299.

[33] Gerszten PC, Burton SA, Welch WC, Brufsky AM, Lembersky BC, Ozhasoglu C, et al. Single-fraction radiosurgery for the treatment of spinal breast metastases. Cancer 2005;104:2244-54. doi:10.1002/cncr.21467.

[34] Pichon B, Thillays F, Bourgier C, Mahé M-A, Supiot S. Radiothérapie stéréotaxique hypofractionnée des métastases osseuses. Cancer Radiother 2014;18:342-9. doi:10.1016/j.canrad.2014.04.005.

[35] Katsoulakis E, Riaz N, Cox B, Mechalakos J, Zatcky J, Bilsky M, et al. Delivering a third course of radiation to spine metastases using image-guided, intensity-modulated radiation therapy. $\mathrm{J}$ Neurosurg Spine 2013;18:63-8. doi:10.3171/2012.9.SPINE12433.

[36] Sellin JN, Reichardt W, Bishop AJ, Suki D, Rhines LD, Settle SH, et al. Factors affecting survival in 37 consecutive patients undergoing de novo stereotactic radiosurgery for contiguous sites of vertebral body metastasis from renal cell carcinoma. J Neurosurg Spine 2015;22:52-9. doi:10.3171/2014.9.SPINE1482. 
[37] Haley ML, Gerszten PC, Heron DE, Chang Y-F, Atteberry DS, Burton SA. Efficacy and costeffectiveness analysis of external beam and stereotactic body radiation therapy in the treatment of spine metastases: a matched-pair analysis. J Neurosurg Spine 2011;14:537-42. doi:10.3171/2010.12.SPINE10233.

[38] Kim H, Rajagopalan MS, Beriwal S, Huq MS, Smith KJ. Cost-effectiveness analysis of single fraction of stereotactic body radiation therapy compared with single fraction of external beam radiation therapy for palliation of vertebral bone metastases. Int J Radiat Oncol 2015;91:556-63. doi:10.1016/j.ijrobp.2014.10.055.

[39] Bijlani A, Aguzzi G, Schaal DW, Romanelli P. Stereotactic radiosurgery and stereotactic body radiation therapy cost-effectiveness results. Front Oncol 2013;3:77. doi:10.3389/fonc.2013.00077. 
Tableau 1. Étude rétrospective monocentrique sur le coût de la radiothérapie des métastases osseuses en France : caractéristiques des patients et des traitements.

Monocentric retrospective study on the cost of radiotherapy for bone metastases in France: characteristics of patients and treatments.

\begin{tabular}{|c|c|c|c|c|c|}
\hline & 8 Gy & $20 \mathrm{~Gy}$ & $30 \mathrm{~Gy}$ & Stéréotaxie & Total \\
\hline Patients (n) & 15 & 21 & 29 & 26 & 91 \\
\hline \multicolumn{6}{|l|}{ Sexe $(n)$} \\
\hline Homme & 10 & 11 & 13 & 10 & $44(48 \%)$ \\
\hline Femme & 5 & 10 & 16 & 16 & $47(52 \%)$ \\
\hline \multicolumn{6}{|l|}{ Age (ans) } \\
\hline Médiane & 63 & 64 & 65 & 60 & 63 \\
\hline Intervalle & $25-83$ & $43-87$ & $47-88$ & $42-87$ & $25-88$ \\
\hline \multicolumn{6}{|l|}{ Cancer primitif $(\mathrm{n})$} \\
\hline Sein & 3 & 4 & 11 & 14 & $32(35 \%)$ \\
\hline Poumon & 3 & 5 & 5 & 2 & $15(16 \%)$ \\
\hline Prostate & 2 & 3 & 2 & 4 & $11(12 \%)$ \\
\hline Voies aérodigestives supérieures & 1 & 3 & 3 & 0 & $7(8 \%)$ \\
\hline Esophage & 2 & 1 & 3 & 0 & $6(7 \%)$ \\
\hline Digestif & 0 & 1 & 2 & 3 & $6(7 \%)$ \\
\hline Autres & 4 & 4 & 3 & 3 & $14(15 \%)$ \\
\hline $\begin{array}{l}\text { Métastases viscérales (nombre de } \\
\text { patients) }\end{array}$ & 11 & 11 & 18 & 13 & $53(58 \%)$ \\
\hline Pulmonaires & 7 & 7 & 9 & 8 & 31 \\
\hline Hépatiques & 5 & 5 & 10 & 6 & 26 \\
\hline Cérébrales & 2 & 4 & 3 & 0 & 9 \\
\hline Ganglionnaires & 6 & 6 & 13 & 4 & 29 \\
\hline Autres & 6 & 5 & 6 & 3 & 20 \\
\hline \multicolumn{6}{|c|}{ Nombre de localisation métastatiques osseuses (n) } \\
\hline 1 & 2 & 1 & 3 & 11 & 17 \\
\hline 2 & 0 & 1 & 1 & 2 & 4 \\
\hline 3 & 4 & 2 & 2 & 3 & 11 \\
\hline 4 & 0 & 2 & 2 & 2 & 6 \\
\hline 5 & 0 & 0 & 1 & 1 & 2 \\
\hline$>5$ & 9 & 15 & 20 & 7 & 51 \\
\hline Antécédents de radiothérapie osseuse (n) & 0 & 5 & 9 & 10 & $24(26 \%)$ \\
\hline \multicolumn{6}{|l|}{ Distance domicile-centre de soins $(\mathrm{km})$} \\
\hline Médiane & 45 & 26 & 38 & 42 & 35 \\
\hline Intervalle & $1-327$ & $1-169$ & $1-130$ & $3-167$ & $1-327$ \\
\hline \multicolumn{6}{|l|}{ Mode de transport } \\
\hline Véhicule sanitaire léger & 8 & 12 & 18 & 14 & 52 \\
\hline Ambulance & 1 & 1 & 2 & 0 & 4 \\
\hline Véhicule personnel & 1 & 1 & 1 & 8 & 11 \\
\hline Transport en commun & 0 & 1 & 2 & 2 & 5 \\
\hline
\end{tabular}




\begin{tabular}{|c|c|c|c|c|c|}
\hline Transport interne & 4 & 6 & 5 & 1 & 16 \\
\hline Autre & 1 & 0 & 1 & 1 & 3 \\
\hline $\begin{array}{l}\text { Hospitalisation au cours de l'irradiation } \\
\text { (n) }\end{array}$ & 4 & 7 & 8 & 2 & $21(23 \%)$ \\
\hline $\begin{array}{l}\text { Nombre de patients avec deux ou trois } \\
\text { traitements concomitants }\end{array}$ & 3 & 5 & 8 & 7 & 23 \\
\hline \multicolumn{6}{|l|}{ Score selon l'OMS préthérapeutique } \\
\hline Médiane & 2 & 2 & 1 & 1 & 1 \\
\hline Intervalle & $0-4$ & $0-4$ & 01-mars & $0-2$ & $0-4$ \\
\hline \multicolumn{6}{|l|}{ Indice de Karnofsky préthérapeutique } \\
\hline Médiane & $70 \%$ & $70 \%$ & $80 \%$ & $100 \%$ & $80 \%$ \\
\hline Intervalle & $\begin{array}{l}60 \%- \\
100 \%\end{array}$ & $\begin{array}{l}40 \%- \\
100 \%\end{array}$ & $\begin{array}{l}60 \%- \\
90 \%\end{array}$ & $60 \%-100 \%$ & $40 \%-100 \%$ \\
\hline \multicolumn{6}{|c|}{ Douleur préthérapeutique (score selon l'échelle visuelle analogique) } \\
\hline Médiane & 8 & 8 & 6 & 0 & 6 \\
\hline Intervalle & 06-oct & $0-10$ & $0-9$ & $0-9$ & $0-10$ \\
\hline \multicolumn{6}{|c|}{ Score selon l'OMS après radiothérapie immédiate } \\
\hline Médiane & 2 & 2 & 1 & 0 & 1 \\
\hline Intervalle & $0-4$ & 01-avr & 01-mars & $0-3$ & $0-4$ \\
\hline \multicolumn{6}{|c|}{ Indice de Karnofsky après radiothérapie immédiate } \\
\hline Médiane & $70 \%$ & $70 \%$ & $80 \%$ & $100 \%$ & $80 \%$ \\
\hline Intervalle & $\begin{array}{l}60 \%- \\
90 \%\end{array}$ & $\begin{array}{l}50 \%- \\
90 \%\end{array}$ & $\begin{array}{l}50 \%- \\
90 \%\end{array}$ & $50 \%-100 \%$ & $50 \%-100 \%$ \\
\hline \multicolumn{6}{|c|}{ Douleur après radiothérapie immédiate (score selon l'échelle visuelle analogique) } \\
\hline Médiane & 6 & 4 & 3 & 0 & 3 \\
\hline Intervalle & $0-10$ & $0-10$ & $0-10$ & $0-7$ & $0-10$ \\
\hline Réirradiation (n) & 5 & 2 & 0 & 2 & 9 \\
\hline $\begin{array}{l}\text { Autres localisations métastatiques } \\
\text { osseuses irradiées postérieurement } \\
\text { pendant la période de suivi (n) }\end{array}$ & 2 & 3 & 6 & 6 & 17 \\
\hline \multicolumn{6}{|l|}{ Décès $(n)$} \\
\hline$<\mathrm{M} 1$ & 2 & 2 & 4 & 0 & 8 \\
\hline M1M2 & 2 & 3 & 2 & 0 & 7 \\
\hline M2M3 & 1 & 4 & 0 & 1 & 6 \\
\hline M3M6 & 1 & 0 & 5 & 2 & 8 \\
\hline$>\mathrm{M} 6$ & 8 & 6 & 9 & 3 & 26 \\
\hline Total & 14 & 15 & 20 & 6 & 55 \\
\hline
\end{tabular}

OMS : Organisation mondiale de la santé ; M : mois. 
Tableau 2. Étude rétrospective monocentrique sur le coût de la radiothérapie des métastases osseuses en France : coûts des traitements et du suivi (en euros).

Monocentric retrospective study on the cost of radiotherapy for bone metastases in France: costs of treatments and follow-up (in euros).

\begin{tabular}{|c|c|c|c|c|c|}
\hline & $8 \mathrm{~Gy}$ & $20 \mathrm{~Gy}$ & $30 \mathrm{~Gy}$ & Stéréotaxie & Total \\
\hline Nombre de patients & 15 & 21 & 29 & 26 & 91 \\
\hline \multicolumn{6}{|l|}{ Radiothérapie } \\
\hline Total & 16096 & 32346 & 72984 & 141196 & 262622 \\
\hline Moyenne & 1071 & 1540 & 2517 & 5430 & 2886 \\
\hline Médiane & 1134 & 1677 & 2358 & 5239 & 2341 \\
\hline Intervalle & $135-2167$ & $272-2676$ & $952-5556$ & $4116-7380$ & $135-7380$ \\
\hline \multicolumn{6}{|l|}{ Transport } \\
\hline Total & 1175 & 6277 & 22466 & 6164 & 36083 \\
\hline Dont franchise & 36 & 236 & 740 & 160 & 1172 \\
\hline Médiane & 39 & 216 & 589 & 163 & 197 \\
\hline Intervalle & $0-257$ & $0-1603$ & $0-2281$ & $0-743$ & $0-2281$ \\
\hline \multicolumn{6}{|l|}{ Réirradiation (total) } \\
\hline Nombre & 5 & 2 & 0 & 2 & 9 \\
\hline $0-1$ mois & 1350 & 0 & 0 & 0 & 1350 \\
\hline $0-3$ mois & 1350 & 0 & 0 & 0 & 1350 \\
\hline $0-6$ mois & 4583 & 810 & 0 & 0 & 5393 \\
\hline$\geq 6$ mois & 4718 & 810 & 0 & 3475 & 9003 \\
\hline \multicolumn{6}{|c|}{ Radiothérapies suivantes sur d'autres localisations métastatiques osseuses (total) } \\
\hline $0-1$ mois & 1134 & 0 & 0 & 6338 & 7472 \\
\hline 0-3 mois & 2494 & 4640 & 0 & 11577 & 18711 \\
\hline $0-6$ mois & 2494 & 4640 & 4068 & 11577 & 22779 \\
\hline$\geq 6$ mois & 4835 & 4640 & 11266 & 28349 & 49090 \\
\hline \multicolumn{6}{|l|}{ Suivi (total) } \\
\hline 0-1 mois & 52797 & 103259 & 89078 & 14733 & 259732 \\
\hline $0-3$ mois & 75225 & 161400 & 151098 & 48680 & 436403 \\
\hline 0-6 mois & 111163 & 250101 & 238083 & 67612 & 666959 \\
\hline
\end{tabular}

